\title{
Simple Robust Normalized PI Control for Controlled Objects with One-order Modelling Error
}

\author{
Makoto Katoh \\ Osaka Institute of Technology \\ Japan
}

\section{Introduction}

In this section, the small gain theorem is introduced as a backgnound theory of this chapter. Then, a lange mission on safety and a small mission on analytic solutions ane introduced after indicating the some problems in discussing nobust PI control systems. Moneover, the way how it came to be possible to obtain the analytic solution of PI control adjustment for the concrete robust control problems with uncertain modeling error which is impossible using the space theory for MIMO systems, is shown for a SISO system. The worst lines of closed loop gain margin were shown in a parameter plane. Finally, risk, merit and demerit of the robust control is discussed and the countermeasure for safeness of that is introduced. And some theme, eg., in the lag time system, the MIMO system and a class of non-linear system for expansion of the approach of this chapter is introduced.

- Many researchers have studied on many kinds of robust system recently. The basic robust stability concept is based on the small gain theorem (Zbou K. with Doyle F. C. and Glover K., 1996). The theorem insists that a closed loop system is internal (robust) stable sufficiently and necessary if the $H_{\infty}$ norm of the nominal closed loop transfer function is smaller than the inverse of $H_{\infty}$ norm of the any uncertainty of feedback elements. (Fig. 1) Moreover, the expansion of the theorem claims that a closed loop system is stable sufficiently if the product of $H_{\infty}$ norms of open loop transfer functions is smaller than 1 when the forward and the feedback transfer functions are both stable.

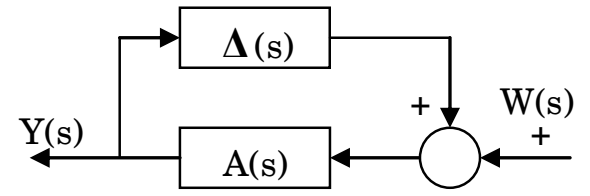

when $\|\Delta\|_{\infty} \leq \gamma$ if $\|\mathrm{A}\|_{\infty}<\frac{1}{\gamma}$ then internal stable

Fig. 1. Feed back system configuration with unknown feedback element

- In MIMO state space models (A,B,C,D), a necessary and sufficient condition using LMI (Linear Matrix Inequality) for the above bounded norm of controlled objects is known as the following Bounded Real Lemma (Zhou K. And Khargonekar P.P., 1988) using the Riccati unequality and Shure complement. 


$$
\exists P=P^{T}>0 \text { such that }\left[\begin{array}{ccc}
P A+A^{T} P & P B & C^{T} \\
B^{T} P & -\frac{1}{\gamma} I_{m} & D^{T} \\
C & D & -\frac{1}{\gamma} I_{p}
\end{array}\right]<0 \Leftrightarrow\|G(s)\|_{\infty}<\frac{1}{\gamma}
$$

A gain margin between the critical closed loop gain of a dependent type IP controller by the Furwits criteria and the analytical closed loop gain solution when closed loop Hardy space norm became 1, and the parametric stability margin (Bhattacharyya S. P., Chapellat H., and Keel L. H., 1994; Katoh 2010) on uncertain time constant and damping coefficient were selected in this chapter for its easiness and robustness although it was expected also using this lemma that internal stable concrete conditions for controlled objects and forward controllers may obtain.

- One of $H_{\infty}$ control problems is described to obtain a robust controller $\mathrm{K}(\mathrm{s})$ when Hardy space norm of closed loop transfer function matrix is bounded like Fig.2 assuming various (additive, multiplicative, left co-prime factor etc.) uncertainty of controlled objects P(s) (Zbou K. with Doyle F. C. and Glover K., 1996).

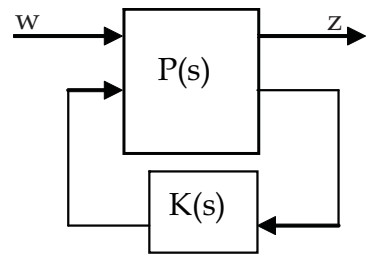

$$
\begin{aligned}
& \mathbf{z}=\boldsymbol{\Phi} \mathbf{w} \\
& \mathbf{\Phi}=\mathbf{P}_{11}+\mathbf{P}_{12} \mathbf{K}\left(\mathbf{I}-\mathbf{P}_{22} \mathbf{K}\right)^{-\mathbf{1}} \mathbf{P}_{21},\|\boldsymbol{\Phi}\|_{\infty}<\gamma
\end{aligned}
$$

Fig. 2. Feed back system configuration for obtained robust control K(s) when Hardy space norm of closed loop transfer function matrix is bounded

- The purpose of this chapter for the robust control problem is to obtain analytical solution of closed loop gain of a dependent type IP controller and analyze robustness by closed loop gain margin for $2^{\text {nd }}$ order controlled objects with one-order feedback like (left co-prime factor) uncertainty as Fig.1 in some tuning regions of IP controller when Hardy space norm of closed loop transfer function matrix is bounded less than 1 .

- Though another basic robust problem is a cooperation design in frequency region between competitive sensitivity and co-sensitivity function, it was omitted in this chapter because a tuning region of IP control was superior for unknown input disturbance other tuning region was superior for unknown reference disturbance.

- However, there is some one not simple for using higher order controllers with many stable zeros and using the norm with window (Kohonen T., 1995, 1997) for I in Hardy space for evaluating the uncertainty of models. Then, a number of robust PI or PID controller and compensator design methods have recently been proposed. But, they are not considered on the modelling error or parameter uncertainty.

- Our given large mission is to construct safe robust systems using simple controllers and simple evaluating method of the uncertainty of models. Then, we have proposed robust PI controllers for controlled objects without stable zeros (Katoh M., 2008, 2009). Our small mission in this chapter is to obtain analytical solution of controller gain with flat 
gain curve in a band width as Butter-worse filter for the $3^{\text {rd }}$ order closed systems with one-order modelling errors and to show the robust property by loop gain margin for damping coefficients of nominal controlled objects and time constants of missing objects (sensor and signal conditioner) using Table Computation Tool (Excel: Microsoft Co. LTD). It is interesting and important historically that infinity time constant is contained in the investing set though it isn't existing actually. Moreover, we confirm the robustness for a parameter change by raising and lowering of step response using CAD Tool (Simulink: Mathworks Co. LTD).

- Risk of Integral term of PI controller when disconnecting the feedback line can be rescued by $\mathrm{M} / \mathrm{A}$ station used in many industrial applications or by shutdown of the plant in our standing point. Then, we show a simple soft M/A station for simulation with PI controllers in appendix.

- This method is not actually because it becomes complicated to computation for higher order objects contained plants with lag time as pointed out in appendix but useful.

\section{System description}

In this section, a description of the higher onder generalized system for later $2^{\text {nd }}$ onder examples with one-onder modeling error is presented although they may not computed concretely.

\subsection{Normalized transfer function}

In this section, how to normalize and why to normalize transfer functions are explained.

The following transfer functions of controlled objects Eq. (1) with multiplicative one-order modeling error Eq. (2) are normalized using a general natural angular frequency $\omega_{n}^{*}$ and gain $K^{*}=K_{o} K_{s}$ as Eq. (3) although the three positions distributed for normalization are different.

$$
\begin{gathered}
G(s)=K_{o} \prod_{i=1}^{r} \frac{\omega_{n i}{ }^{2}}{s^{2}+2 \varsigma_{i} \omega_{n i} s+\omega_{n i}{ }^{2}} \prod_{j=1}^{q} \frac{\alpha_{j}}{s+\alpha_{j}} \\
H(s)=\frac{K_{s}}{\varepsilon s+1} \\
\bar{G}_{l}(s)=\frac{1}{K^{*}} G(s) H(s) \\
=\frac{\omega_{n}^{* 2 r+q+1}}{\varepsilon s^{2 r+q+1}+\beta_{2 r+q} \omega_{n}^{*} s^{2 r+q}+\cdots+\beta_{1} \omega_{n}^{* 2 r+q} s+\omega_{n}^{* 2 r+q+1}}
\end{gathered}
$$

Moreover, converting the differential operator s to $\bar{s}$ as,

$$
\bar{s} \triangleq \frac{s}{\omega_{n}^{*}}, \bar{\varepsilon}=\omega_{n}^{*} \varepsilon
$$

the following normalized open loop transfer function is obtained:

$$
\bar{G}_{l}(\bar{s})=\frac{1}{\bar{\varepsilon} \bar{s}^{n+1}+\bar{\beta}_{2 r+q} \bar{s}^{n}+\cdots+\bar{\beta}_{1} \bar{s}+1} \text { where } n=2 r+q
$$


Neglecting one-order modeling error, the following normalized open loop transfer function is obtained:

$$
\bar{G}(\bar{s})=\frac{1}{\bar{s}^{n}+\gamma_{n-1} \bar{s}^{n-1} \cdots+\gamma_{1} \bar{s}+1} \text { where } \mathrm{n}=2 \mathrm{r}+\mathrm{q}
$$

\subsection{State space models}

In this section, 3 kinds of description on normalized state space models are shown although they may not computed concretely. First shows a continuous realization form of the higher onder transfer functions to a SISO system. Second shows a normalized sampled system form for the first continuous realization on sampling points. Thind shows a normalized continuously approximated form using logarithm conversion for the second sampled system.

Minimum realization of normalized transfer function: The normalized transfer function, shown in Eq. (6), is converted to the following SISO controllable minimum realization:

$$
\begin{aligned}
& \dot{\mathbf{x}}(t)=\tilde{\mathbf{A}} \mathbf{x}(t)+\tilde{\mathbf{b}} u(t) \\
& y(t)=\tilde{\mathbf{c}} \mathbf{x}(t)+d u(t)
\end{aligned}
$$

Normalized sampled system on sampling points: Integrating the response between two sampling points to the next sampling point, the following precise sampled system is obtained:

$$
\begin{aligned}
& \mathbf{x}((k+1) h)=e^{\tilde{\mathbf{A}} h} \mathbf{x}(k h)-\tilde{\mathbf{A}}^{-1}\left[\mathbf{I}-e^{\tilde{\mathbf{A}} h}\right] \tilde{\mathbf{b}} u(k h) \\
& y(k h)=\tilde{\mathbf{c}} \mathbf{x}(k h)+\tilde{d} u(k h)
\end{aligned}
$$

Normalized sampled system approximated:

Approximating Eq. (3) by the advanced difference method, the following sampled system is obtained:

$$
\begin{aligned}
& \mathbf{x}(k+1)=(\mathbf{I}+\tilde{\mathbf{A}} h) \mathbf{x}(k)+\tilde{\mathbf{b}} h u(k) \\
& y(k)=\tilde{\mathbf{c}} \mathbf{x}(k)+d u(k)
\end{aligned}
$$

Normalized System in continuous region:

Returning to the continuous region after conversion using the matrix logarithm function, the following system is obtained in continuous region:

$$
\begin{aligned}
\dot{\mathbf{x}}(t) & =\mathbf{A}^{*} \mathbf{x}(t)+\mathbf{b}^{*} u(t) \\
y(t) & =\tilde{\mathbf{c}} \mathbf{x}(t)+\tilde{d} u(t) \\
\text { where } \quad \mathbf{A}^{*} & =\frac{1}{h} \ln (\mathbf{I}+\tilde{\mathbf{A}} h) \\
& =\tilde{\mathbf{A}}-\tilde{\mathbf{A}}^{2} h+\frac{1}{2} \tilde{\mathbf{A}}^{3} h^{2} \cdots \\
\mathbf{b}^{*} & =\left(\mathbf{I}-\tilde{\mathbf{A}} h+\frac{1}{2} \tilde{\mathbf{A}}^{2} h^{2} \cdots\right) \tilde{\mathbf{b}}
\end{aligned}
$$


The condition of convergence for logarithm conversion Eq. (11) of controllable accompany description Eq. (7) is not described because it is assumed that the sampled time $h$ is sufficiently small. The approximated order is then selected as the $9^{\text {th }}$ order. Thus, $\tilde{d}=0$ is assumed for the simplification.

\section{Controller and parameter tuning}

In this section, an IP controller and a number of parameter tuning methods are presented in onder to increase the robustness of the control system.

3.1 Normalized IP controller

In this section, 3 kinds of description on normalized integral lead dependent type IP controller which is not conventional proportional lead dependent type PI controller are shown. First is showing inherent frequency for normalization as magnitudes of integnal and proportional in continuous systems. Second is showing that in digital systems. Thind is showing again that of digital systems in returning approximated continuous systems.

$$
\begin{gathered}
C(\bar{s})=\bar{K}_{i}\left(\frac{1}{\bar{s}}+\bar{p}\right)=\bar{K}_{i} \omega_{n}\left(\frac{1}{s}+\frac{\bar{p}}{\omega_{n}}\right) \\
C^{*}(\bar{z})=\frac{\bar{K}_{i} \bar{p}(\bar{z}-1+\bar{h} / \bar{p})}{\bar{z}-1}=\frac{\bar{K}_{i} \bar{p}\left(z-1+h \omega_{n}^{*} / \bar{p}\right)}{z-1} \\
C^{*}(\bar{s})=\bar{K}_{i}\left(\frac{1}{\bar{s}}+\bar{p}\right)=\bar{K}_{i} \omega_{n}^{*}\left(\frac{1}{s}+\frac{\bar{p}}{\omega_{n}^{*}}\right)
\end{gathered}
$$

Note that the digital IP controller of Eq. (13) is asymptotic to the proportional control as $h$ approaches zero or $\bar{p}$ becomes larger. This controller is called IPL tuning. Then, the stable zero $=-1 / \bar{p}$ must be placed not in the neighborhood of the system poles for safety.

\subsection{Stability of closed loop transfer function}

In this section, mone higher onder systems are processed for consideration genenally on three tuning region classified by the amplitude of $P$ control parameter using Hurwits approach in example of a second-onder system with one-onder modelling error. It is guessed that there may be four elementary tuning regions and six combinatorial tuning regions generally in the aspect of Hurwits stability.

The following normalized loop transfer function is obtained from the normalized controlled object Eq. (5) and the normalized controller Eq. (12):

$$
W(\bar{s})=\frac{\bar{K}_{i}(1+\overline{p s})(\bar{\varepsilon} \bar{s}+1)}{\bar{\varepsilon} \bar{s}^{n+2}+\bar{\beta}_{2 r+q} \bar{s}^{n+1}+\cdots+\bar{\beta}_{1} \bar{s}^{2}+\left(\bar{K}_{i} \bar{p}+1\right) \bar{s}+\bar{K}_{i}}
$$

If the original parameters $\forall i, j, \varsigma_{i}>0, \alpha_{j}>0$ are positive, then $\forall k, \beta_{k}>0$. Assuming $\bar{p}>0$ and $\bar{K}_{i}>0$, and that

$$
\varphi(\bar{s}) \triangleq \varepsilon \bar{s}^{n+2}+\beta_{2 r+q} \bar{s}^{n+1}+\cdots+\beta_{1} \bar{s}^{2}+\left(\bar{K}_{i} \bar{p}+1\right) \bar{s}+\bar{K}_{i}
$$


is a Hurwits polynomial, the stability limits of $\bar{K}_{i}$ can be obtained as a region of $\bar{p}$. Then, this region is called a IPL region when $\bar{p}$ has a maximum lower bound and an IPO region when $\bar{p}=0$. The region between zero and the minimum upper bound is called the IPS. The region between the minimum upper bound and the maximum lower bound is called the IPM region. Generally, there are four elementary regions and six combinatorial regions.

\subsection{Stationary points investing approach on fraction equation}

In this section, Stationary Points Investing approach on Fraction Equation for seanching local maximum with equality restriction is shown using Lagrange's undecided multiplier approach. Then, multiple same solutions of the independent variable are solved at the stationary points. They can be used to check for mistakes in calculation as self-diagnostics approach.

Here, the common normalized control parameters $\bar{K}_{i}$ and $\bar{p}$ will be obtained in continuous region, which has reduction models reduced from original region.

Stationary Points Investing for Fraction Equation approach for seanching local maximum with equality restriction:

$$
\begin{aligned}
& \qquad|W(j \bar{\omega})|=\left[\frac{u(\bar{\omega})}{v(\bar{\omega})}\right]^{0.5} \\
& \rightarrow \text { solve_local_muximum/minimum for } \quad \bar{\omega}=\bar{\omega}_{s} \\
& \text { such that }\left|W\left(j \bar{\omega}_{s}\right)\right|=1
\end{aligned}
$$

This is the design policy of servo control for wide band width. In particular, $|W(o)|=1$ means that steady state error is 0 .

Next, Lagrange's undecided multiplier approach is applied to obtain stationary points $\bar{\omega}_{s}$ with equality restriction using the above $u, v$ notations.

Then, the original problem can be converted to the following problem:

$$
\begin{gathered}
J^{+}(\bar{\omega}, \lambda)=|W(j \bar{\omega})|^{2+}=\frac{u(\bar{\omega})}{v(\bar{\omega})}+\lambda\{u(\bar{\omega})-v(\bar{\omega})\} \\
\rightarrow \text { solve_local_maximum/minimum }
\end{gathered}
$$

where $\lambda$ is a Lagrange multiplier.

The necessary conditions for obtaining the local minimum/maximum of a new function become as followings.

$$
\begin{gathered}
\left.\frac{\partial J^{+}(\bar{\omega}, \lambda)}{\partial \bar{\omega}}\right|_{\bar{\omega}=\bar{\omega}_{s}}=\frac{u^{\prime}\left(\bar{\omega}_{s}\right) v\left(\bar{\omega}_{s}\right)-u\left(\bar{\omega}_{s}\right) v^{\prime}\left(\bar{\omega}_{s}\right)}{v\left(\bar{\omega}_{s}\right)^{2}}+\lambda\left\{u{ }^{\prime}\left(\bar{\omega}_{s}\right)-v^{\prime}\left(\bar{\omega}_{s}\right)\right\}=0 \\
\left.\frac{\partial J^{+}(\bar{\omega}, \lambda)}{\partial \lambda}\right|_{\bar{\omega}=\bar{\omega}_{s}}=u\left(\bar{\omega}_{s}\right)-v\left(\bar{\omega}_{s}\right)=0
\end{gathered}
$$

The following relations are obtained from eqs. (19) and (20): 


$$
\begin{aligned}
& u^{\prime}\left(\bar{\omega}_{s}\right)=v^{\prime}\left(\bar{\omega}_{s}\right) \text { or } \quad \lambda=-\frac{1}{v\left(\bar{\omega}_{s}\right)} \\
& u\left(\bar{\omega}_{s}\right)=v\left(\bar{\omega}_{s}\right)
\end{aligned}
$$

Solutions of control parameters:

Solving these simultaneous equations, the following functions can be obtained:

$$
\begin{aligned}
& \overline{\mathbf{\omega}}_{\mathbf{s}}=\mathbf{f}(\mathbf{a}, \bar{p}) \\
& \bar{K}_{i}^{j}=g\left(\mathbf{a}, \bar{p}, \bar{\omega}_{s j}\right) \quad(j=1,2, \cdots, \alpha)
\end{aligned}
$$

where $\bar{\omega}_{s}$ is the stationary points vector.

Multiple solutions of $\bar{K}_{i}$ can be used to check for mistakes in calculation.

3.4 Example of a second-order system with one-order modelling error In this section, an IP control system in continuous design for a second-onder original controlled object without one-onder sensor and signal conditioner dynamics is assumed for simplicity. The closed loop system with uncertain one-onder modeling error is normalized and obtained the stable region of the integral gain in the three tuning region classified by the amplitude of $P$ control parameter using Hurwits approach. Then, the safeness of the only I tuning region and the risk of the large P tuning region are discussed. Moneover, the analytic solutions of stationary points and double same integral gains ane obtained using the Stationary Points Investing on Fraction Equation approach for the gain curve of a closed loop system.

Here, an IP control system for a second-order controlled object without sensor dynamics is assumed.

Closed-loop transfer function:

$$
\begin{gathered}
G(s)=\frac{K_{o} \omega_{n}^{2}}{s^{2}+2 \varsigma \omega_{n} s+\omega_{n}^{2}} \\
H(s)=\frac{K_{s}}{\varepsilon s+1} \\
\bar{G}_{o}(s)=\frac{1}{K_{s} K_{o}} G(s) H(s)=\frac{\omega_{n}^{2}}{(\varepsilon s+1)\left(s^{2}+2 \varsigma \omega_{n} s+\omega_{n}^{2}\right)} \\
\bar{s} \triangleq \frac{s}{\omega_{n}}, \bar{\varepsilon}=\omega_{n} \varepsilon \\
W(\bar{s})=\frac{\bar{K}_{i}(1+\overline{p s})(\overline{\varepsilon s}+1)}{\bar{\varepsilon} s^{4}+(2 \varsigma \bar{\varepsilon}+1) \bar{s}^{3}+(\bar{\varepsilon}+2 \varsigma) \bar{s}^{2}+\left(\bar{K}_{i} \bar{p}+1\right) \bar{s}+\bar{K}_{i}}
\end{gathered}
$$

Stable conditions by Hurwits approach with four parameters:

a. In the case of a certain time constant

IPL\&IPS Common Region: 


$$
\begin{gathered}
0<\bar{K}_{i}<\max \left[0, \min \left[\bar{k}_{2}, \bar{k}_{3}, \infty\right]\right] \\
\bar{k}_{2} \triangleq \frac{2 \varsigma\left(\bar{\varepsilon}^{2}+2 \varsigma \bar{\varepsilon}+1\right)}{\bar{\varepsilon} \bar{p}} \\
{\left[\bar{p}\left\{4 \varsigma^{2} \bar{\varepsilon}+2 \varsigma \bar{\varepsilon}^{2}+2 \varsigma-\bar{\varepsilon}\right\}-(2 \varsigma \bar{\varepsilon}+1)^{2}\right]} \\
\bar{k}_{3} \triangleq \sqrt{\frac{\left[\bar{p}\left\{4 \varsigma^{2} \bar{\varepsilon}+2 \varsigma \bar{\varepsilon}^{2}+2 \varsigma-\bar{\varepsilon}\right\}-(2 \varsigma \bar{\varepsilon}+1)^{2}\right]^{2}}{+8 \bar{\varepsilon} \varsigma \bar{p}^{2}\left(\bar{\varepsilon}^{2}+2 \varsigma \bar{\varepsilon}+1\right)}} \frac{2 \bar{\varepsilon} \bar{p}^{2}}{}
\end{gathered}
$$

where $\bar{p}>0$ for $4 \varsigma^{2} \bar{\varepsilon}+2 \varsigma \bar{\varepsilon}^{2}+2 \varsigma \leq \bar{\varepsilon}$

\section{IPL, IPS Separate Region:}

The integral gain stability region is given by Eqs. (28)-(30).

$$
\begin{aligned}
& 0<\frac{(2 \varsigma \bar{\varepsilon}+1)^{2}}{4 \varsigma^{2} \bar{\varepsilon}+2 \bar{\varepsilon}^{2}+2 \varsigma-\bar{\varepsilon}} \leq \bar{p} \\
& 0<\bar{p}<\frac{(2 \varsigma \bar{\varepsilon}+1)^{2}}{4 \varsigma^{2} \bar{\varepsilon}+2 \varsigma^{2}+2 \varsigma-\bar{\varepsilon}} \\
& \text { for } \quad 4 \varsigma^{2} \bar{\varepsilon}+2 \bar{\varepsilon}^{2}+2 \varsigma-\bar{\varepsilon}>0
\end{aligned}
$$

It can be proven that $\bar{k}_{3}>0$ in the IPS region, and

$$
k_{2} \rightarrow \infty, k_{3} \rightarrow \infty \quad \text { when } \quad \bar{p} \rightarrow 0
$$

\section{IPO Region:}

$$
0<\bar{K}_{i}<\frac{2 \varsigma\left(\bar{\varepsilon}^{2}+2 \varsigma \bar{\varepsilon}+1\right)}{(2 \varsigma \bar{\varepsilon}+1)^{2}} \quad \text { where } \quad \bar{p}=0
$$

The IP0 region is most safe because it has not zeros.

b. In the case of an uncertain positive time constant

\section{IPL\&IPS Common Region:}

$$
0<\bar{K}_{i}<\max \left[0, \min \left[\left.\bar{k}_{2}\right|_{\bar{\varepsilon}=1},\left.\bar{k}_{3}\right|_{\bar{\varepsilon}=\bar{\varepsilon}_{p}}\right]\right] \quad \text { when } \bar{p}>0
$$

where $\bar{k}_{3}^{\prime}\left(\bar{p}, \varsigma, \bar{\varepsilon}_{p}\right)=0$

$$
\begin{gathered}
\text { for } \quad 4 \varsigma^{2} \bar{\varepsilon}+2 \varsigma \bar{\varepsilon}^{2}+2 \varsigma<\bar{\varepsilon} \\
\min _{\varepsilon} \bar{k}_{2}=\frac{4 \varsigma(\varsigma+1)}{\bar{p}} \quad \text { when } \quad \bar{\varepsilon}=1
\end{gathered}
$$

\section{IPL, IPS Separate Region:}

This region is given by Eq. (32). 


\section{IPO Region:}

$$
0<\bar{K}_{i}<\left.2 \varsigma\left(1-\varsigma^{2}\right)\right|_{\bar{\varepsilon}=\bar{\varepsilon}_{p}, 0<\varsigma<0.707},\left.\frac{1}{2 \varsigma}\right|_{\overline{\bar{\varepsilon}=+\infty, \varsigma>0.707}}
$$

when $\quad\left(\bar{\varepsilon}_{p}=\frac{\varsigma}{\left(1-2 \varsigma^{2}\right)}>0,0<\varsigma<0.707\right), \bar{p}=0$

c. Robust loop gain margin

The following loop gain margin is obtained from eqs. (28) through (38) in the cases of certain and uncertain parameters:

$$
g m \triangleq \frac{\bar{K}_{i U L}}{\bar{K}_{i}}
$$

where $\bar{K}_{i U L}$ is the upper limit of the stable loop gain $\bar{K}_{i}$.

\section{Stable conditions by Hurwits approach with three parameters:}

The stability conditions will be shown in order to determine the risk of one order modelling error,

$$
\begin{array}{r}
0<\bar{K}_{i} \quad \text { where } \quad \bar{p} \geq \frac{1}{2 \varsigma} \quad(P L) \\
0<\bar{K}_{i}<\frac{2 \varsigma}{1-2 \varsigma \bar{p}} \quad \text { where } 0 \leq \bar{p}<\frac{1}{2 \varsigma}
\end{array}
$$

Hurwits Stability is omitted because $\bar{h}$ is sufficiently small, although it can be checked using the bilinear transform.

\section{Robust loop gain margin:}

$$
g m=\infty \quad\left(P L \_ \text {region }\right)
$$

It is risky to increase the loop gain in the IPL region too much, even if the system does not become unstable because a model order error may cause instability in the IPL region. In the IPL region, the sensitivity of the disturbance from the output rises and the flat property of the gain curve is sacrificed, even if the disturbance from the input can be isolated to the output upon increasing the control gain.

\section{Frequency transfer function:}

$$
\begin{aligned}
& |W(j \bar{\omega})| \triangleq\left[\frac{\bar{K}_{i}^{2}\left\{1+\bar{\omega}^{2} \bar{p}^{2}\right\}}{\left(\bar{K}_{i}-2 \varsigma \bar{\omega}^{2}\right)^{2}+\bar{\omega}^{2}\left\{1-\bar{\omega}^{2}+\bar{K}_{i} \bar{p}\right\}^{2}}\right]^{0.5} \\
& \rightarrow \text { solve_local_maximum/minmum for } \quad \bar{\omega}=\bar{\omega}_{s} \\
& \text { such that }\left|W\left(j \bar{\omega}_{s}\right)\right|=1
\end{aligned}
$$

When the evaluation function is considered to be two variable functions $\left(\bar{\omega}\right.$ and $\left.\bar{K}_{i}\right)$ and the stationary point is obtained, the system with the parameters does not satisfy the above stability conditions. 
Therefore, only the stationary points in the direction of $\bar{\omega}$ will be obtained without considering the evaluation function on $\bar{K}_{i}$ alone.

\section{Stationary points and the integral gain:}

Using the Stationary Points Investing for Fraction Equation approach based on Lagrange's undecided multiplier approach with equality restriction, the following two loop gain equations on $x$ are obtained. Both identities can be used to check for miscalculation.

$$
\begin{aligned}
& \quad \bar{K}_{i 1}=0.5\left\{x^{2}+2\left(2 \varsigma^{2}-1\right) x+1\right\} /\{2 \varsigma+(x-1) \bar{p}\} \\
& \bar{K}_{i 2}=0.5\left\{3 x^{2}+4\left(2 \varsigma^{2}-1\right) x+1\right\} /\{2 \varsigma+(2 x-1) \bar{p}\} \\
& \text { where } \quad x=\bar{\omega}_{s}^{2} \geq 0
\end{aligned}
$$

Equating the right-hand sides of these equations, the third-order algebraic equation and the solutions for semi-positive stationary points are obtained as follows:

$$
x=0, x=\sqrt{\frac{2\left(2 \varsigma^{2}-1\right)(2 \varsigma-\bar{p})}{\bar{p}}-1}
$$

These points, which are called the first and second stationary points, call the first and second tuning methods, respectively, which specify the points for gain 1.

\section{Numerical results}

In this section, the solutions of double same integnal gain for a tuning region at the stationary point of the gain curve of the closed system are shown and checked in some parameter tables on normalized proportional gains and normalized damping coefficients. Moreover, loop gain margins are shown in some parameter tables on uncertain time constants of one-onder modeling error and damping coefficients of original controlled objects for some tuning regions contained with safest only I region.

\begin{tabular}{|l|l|l|l|l|l|}
\hline & 0.9 & 0.95 & 1.00 & 1.05 & 1.10 \\
\hline 0.8 & 0.8612 & 1.0496 & 1.1892 & 1.3068 & 1.4116 \\
\hline 0.9 & 0.6999 & 0.9424 & 1.0963 & 1.2197 & 1.3271 \\
\hline 1.0 & -99 & 0.8186 & 1.0000 & 1.1335 & 1.2457 \\
\hline 1.1 & -99 & 0.6430 & 0.8932 & 1.0446 & 1.1647 \\
\hline 1.2 & -99 & -99 & 0.7598 & 0.3480 & 1.0812 \\
\hline
\end{tabular}

Table 1. $\omega_{p}$ values for $\varsigma$ and $\bar{p}$ in IPL tuning by the first tuning method

\begin{tabular}{|l|l|l|l|l|l|}
\hline & 0.9 & 0.95 & 1.00 & 1.05 & 1.10 \\
\hline 0.8 & 0.7750 & 1.0063 & 1.2500 & 1.5063 & 1.7750 \\
\hline 0.9 & 0.6889 & 0.8944 & 1.1111 & 1.3389 & 1.5778 \\
\hline 1.0 & 1.2272 & 0.8050 & 1.0000 & 1.2050 & 1.4200 \\
\hline 1.1 & 1.1077 & 0.7318 & 0.9091 & 1.0955 & 1.2909 \\
\hline 1.2 & 1.0149 & 1.0791 & 0.8333 & 1.0042 & 1.1833 \\
\hline
\end{tabular}

Table 2. $\bar{K}_{i 1}=\bar{K}_{i 2}$ values for $\varsigma$ and $\mathrm{p}$ in IPL tuning by the first tuning method 
Table 1 lists the stationary points for the first tuning method. Table 2 lists the integration gains $\left(\bar{K}_{i 1}=\bar{K}_{i 2}\right)$ obtained by substituting Eq. (46) into Eqs. (44) and (45) for various damping coefficients.

Table 3 lists the integration gains $\left(\bar{K}_{i 1}=\bar{K}_{i 2}\right)$ for the second tuning method.

\begin{tabular}{|l|l|l|l|l|l|}
\hline & 0.9 & 0.95 & 1.00 & 1.05 & 1.10 \\
\hline 1.3 & 1.0 & 0.8333 & 0.7143 & 0.6250 & 0.5556 \\
\hline 1.4 & 1.250 & 1.0 & 0.8333 & 0.7143 & 0.6250 \\
\hline 1.5 & 1.667 & 1.250 & 1.0 & 0.8333 & 0.7143 \\
\hline 1.6 & 2.50 & 1.667 & 1.250 & 1.0 & 0.8333 \\
\hline 1.7 & 5.00 & 2.50 & 1.667 & 1.250 & 1.0 \\
\hline
\end{tabular}

Table 3. $\bar{K}_{i 1}=\bar{K}_{i 2}$ values for $\varsigma$ and $\bar{p}$ in IPL tuning by the second tuning method

Then, a table of loop gain margins $(g m>1)$ generated by Eq. (39) using the stability limit and the loop gain by the second tuning method on uncertain $\bar{\varepsilon}$ in a given region of $\bar{\varepsilon}$ for each controlled $\varsigma$ by IPL $(\bar{p}=1.5)$ control is very useful for analysis of robustness. Then, the unstable region, the unstable region, which does not become unstable even if the loop gain becomes larger, and robust stable region in which uncertainty of the time constant, are permitted in the region of $\bar{\varepsilon}$.

Figure 3 shows a reference step up-down response with unknown input disturbance in the continuous region. The gain for the disturbance step of the IPL tuning is controlled to be approximately 0.38 and the settling time is approximately 6 sec.

The robustness on indicial response for the damping coefficient change of \pm 0.1 is an advantageous property. Considering Zero Order Hold. with an imperfect dead-time compensator using 1st-order Pade approximation, the overshoot in the reference step response is larger than that in the original region or that in the continuous region.

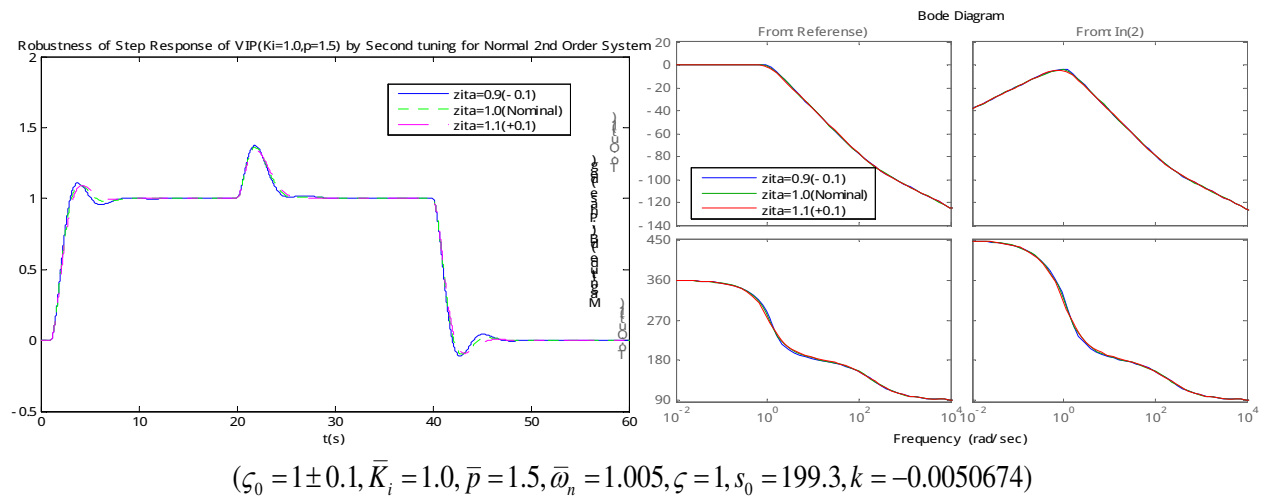

Fig. 3. Robustness of IPL tuning for damping coefficient change.

Then, Table 4 lists robust loop gain margins $(g m>1)$ using the stability limit by Eq.(37) and the loop gain by the second tuning method on uncertain $\bar{\varepsilon}$ in the region of $(0.1 \leq \bar{\varepsilon} \leq 10)$ for each controlled $\varsigma(>0.7)$ by $\operatorname{IPL}(\bar{p}=1.5)$ control. The first gray row shows the area that is also unstable. 
Table 5 does the same for each controlled $\varsigma(>0.4)$ by IPS $(\bar{p}=0.01)$. Table 6 does the same for each controlled $\varsigma(>0.4)$ by $I P 0(\bar{p}=0.0)$.

\begin{tabular}{|r|r||r|r|r|r|r|r|}
\hline eps/zita & 0.3 & 0.7 & 0.8 & 0.9 & 1 & 1.1 & 1.2 \\
\hline 0.1 & -2.042 & -1.115 & 1.404 & 5.124 & 10.13 & 16.49 & 24.28 \\
\hline 0.2 & -1.412 & -0.631 & 0.788 & 2.875 & 5.7 & 9.33 & 13.83 \\
\hline 1.5 & -0.845 & -0.28 & 0.32 & 1.08 & 2 & 3.08 & 4.32 \\
\hline 2.4 & -1.019 & -0.3 & 0.326 & 1.048 & 1.846 & 2.702 & 3.6 \\
\hline 3.2 & -1.488 & -0.325 & 0.342 & 1.06 & 1.8 & 2.539 & 3.26 \\
\hline 5 & -2.128 & -0.386 & 0.383 & 1.115 & 1.778 & 2.357 & 2.853 \\
\hline 10 & -4.596 & -0.542 & 0.483 & 1.26 & 1.81 & 2.187 & 2.448 \\
\hline
\end{tabular}

Table 4. Robust loop gain margins on uncertain $\bar{\varepsilon}$ in each region for each controlled $\varsigma$ at IPL $(\bar{p}=1.5)$

\begin{tabular}{|r|r|r|r|r|r|}
\hline eps/zita & 0.4 & 0.5 & 0.6 & 0.7 & 0.8 \\
\hline 0.1 & 1.189 & 1.832 & 2.599 & 3.484 & 4.483 \\
\hline 0.6 & 1.066 & 1.524 & 2.021 & 2.548 & 3.098 \\
\hline 1 & 1.097 & 1.492 & 1.899 & 2.312 & 2.729 \\
\hline 2.1 & 1.254 & 1.556 & 1.839 & 2.106 & 2.362 \\
\hline 10 & 1.717 & 1.832 & 1.924 & 2.003 & 2.073 \\
\hline
\end{tabular}

Table 5. Robust loop gain margins on uncertain $\bar{\varepsilon}$ in each region for each controlled $\varsigma$ at IPS $(\bar{p}=0.01)$

\begin{tabular}{|r|r|r|r|r|r|r|r|r|}
\hline eps/zita & 0.3 & 0.4 & 0.5 & 0.6 & 0.7 & 0.8 & 0.9 & 1 \\
\hline 0.1 & 0.6857 & 1.196 & 1.835 & 2.594 & 3.469 & 4.452 & 5.538 & 6.722 \\
\hline 0.4 & 0.6556 & 1.087 & 1.592 & 2.156 & 2.771 & 3.427 & 4.118 & 4.84 \\
\hline 0.5 & 0.6604 & 1.078 & 1.556 & 2.081 & 2.645 & 3.24 & 3.859 & 4.5 \\
\hline 0.6 & 0.6696 & 1.075 & 1.531 & 2.025 & 2.547 & 3.092 & 3.655 & 4.231 \\
\hline 1 & 0.7313 & 1.106 & 1.5 & 1.904 & 2.314 & 2.727 & 3.141 & 3.556 \\
\hline 2.1 & 0.9402 & 1.264 & 1.563 & 1.843 & 2.109 & 2.362 & 2.606 & 2.843 \\
\hline 10 & 1.5722 & 1.722 & 1.835 & 1.926 & 2.004 & 2.073 & 2.136 & 2.195 \\
\hline 9999 & 1.9995 & 2 & 2 & 2 & 2 & 2 & 2 & 2 \\
\hline
\end{tabular}

Table 6. Robust loop gain margins on uncertain $\bar{\varepsilon}$ in each region for each controlled $\varsigma$ at IP0 $(\bar{p}=0.0)$

These table data with additional points were converted to the $3 \mathrm{D}$ mesh plot as following Fig. 4. As IP0 and IPS with very small $\bar{p}$ are almost equivalent though the equations differ quiet, the number of figures are reduced. It implies validity of both equations.

According to the line of worst loop gain margin as the parameter of attenuation in the controlled objects which are described by gray label, this parametric stability margin (PSM) (Bhattacharyya S. P., Chapellat H., and Keel L. H., 1994) is classified to 3 regions in IPS and IP0 tuning regions and to 4 regions in IPL tuning regions as shown in Fig.5. We may call the 
larger attenuation region with more than 2 loop gain margin to the strong robust segment region in which region uncertainty time constant of one-order modeling error is allowed in the any region and some change of attenuation is also allowed.

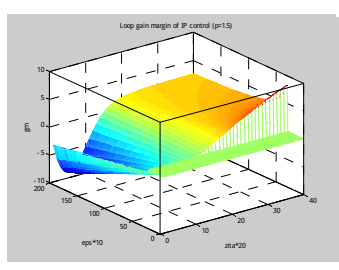

(a) $\bar{p}=1.5$

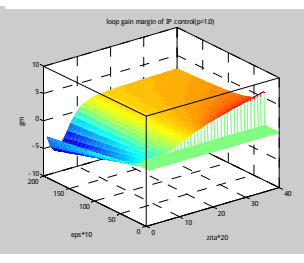

(b) $\bar{p}=1.0$

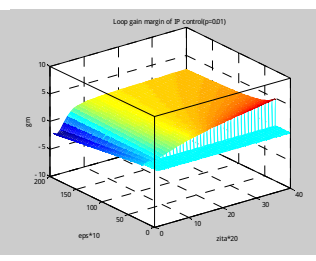

(c) $\bar{p}=0.5$

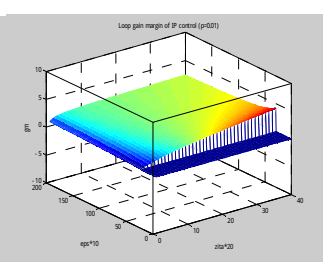

(d) $\bar{p}=0.01$ or 0

Fig. 4. Mesh plot of closed loop gain margin

Next, we call the larger attenuation region with more than $\gamma>1$ and less than 2 loop gain margin to the weak robust segment region in which region uncertainty time constant of one-order modeling error is only allowed in some region over some larger loop gain margin and some larger change of attenuation is not allowed. The third and the forth segment is almost unstable. Especially, notice that the joint of each segment is large bending so that the sensitivity of uncertainty for loop gain margin is larger more than the imagination.

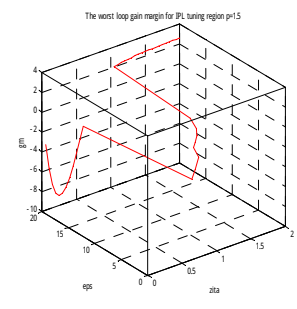

(a) $\bar{p}=1.5$

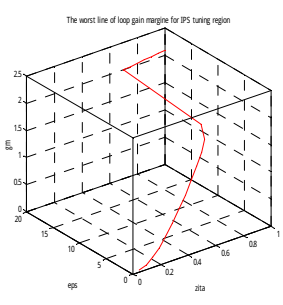

(b) $\bar{p}=0.01$

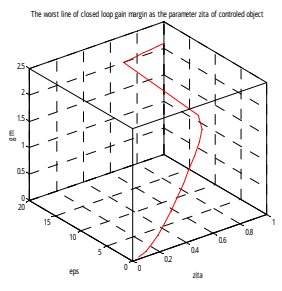

(c) $\bar{p}=0$

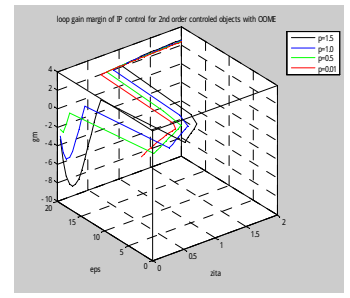

(d) $\bar{p}=1.5,1.0,0.5,0.01$

Fig. 5. The various worst lines of loop gain margin in a parameter plane (certain\&uncertain)

Moreover, the readers had to notice that the strong robust region and weak robust region of IPL is shift to larger damping coefficient region than ones of IPS and IP0. Then, this is also one of risk on IPL tuning region and change of tuning region from IP0 or IPS to IPL region.

\section{Conclusion}

In this section, the way to convert this IP control tuning parameters to independent type PI control is presented. Then, parameter tuning policy and the reason adopted the policy on the controller are presented. The good and no good results, limitations and meanings in this chapter are summarized. The closed loop gain curve obtained from the second onder example with one-onder feedback modeling error implies the butter-worth filter model matching method in higher onder systems may be useful. The Handy space norm with bounded window was defined for I, and nobust stability was discussed for MIMO system by an expanssion of small gain theorem under a bounded condition of closed loop systems. 
- We have obtained first an integral gain leading type of normalized IP controller to facilitate the adjustment results of tuning parameters explaining in the later. The controller is similar that conventional analog controllers are proportional gain type of PI controller. It can be converted easily to independent type of PI controller as used in recent computer controls by adding some converted gains. The policy of the parameter tuning is to make the norm of the closed loop of frequency transfer function contained one-order modeling error with uncertain time constant to become less than 1 . The reason of selected the policy is to be able to be similar to the conventional expansion of the small gain theorem and to be possible in PI control. Then, the controller and uncertainty of the model becomes very simple. Moreover, a simple approach for obtaining the solution is proposed by optimization method with equality restriction using Lagrange's undecided multiplier approach for the closed loop frequency transfer function.

- The stability of the closed loop transfer function was investigated using Hurwits Criteria as the structure of coefficients were known though they contained uncertain time constant.

- The loop gain margin which was defined as the ratio of the upper stable limit of integral gain and the nominal integral gain, was investigated in the parameter plane of damping coefficient and uncertain time constant. Then, the robust controller is safe in a sense if the robust stable region using the loop gain margin is the single connection and changes continuously in the parameter plane even if the uncertain time constant changes larger in a wide region of damping coefficient and even if the uncertain any adjustment is done. Then, IP0 tuning region is most safe and IPL region is most risky.

- Moreover, it is historically and newly good results that the worst loop gain margin as each damping coefficient approaches to 2 in a larger region of damping coefficients.

- The worst loop gain margin line in the uncertainty time constant and controlled objects parameters plane had 3 or 4 segments and they were classified strong robust segment region for more than 2 closed loop gain margin and weak robust segment region for more than $\gamma>1$ and less than 2 loop gain margin. Moreover, the author was presented also risk of IPL tuning region and the change of tuning region.

- It was not good results that the analytical solution and the stable region were complicated to obtain for higher order systems with higher order modeling error though they were easy and primary. Then, it was unpractical.

\section{Appendix}

A. Example of a second-order system with lag time and one-order modelling error

In this section, for applying the robust PI control concept of this chapter to systems with lag time, the systems with one-onder model error are approximated using Pade approximation and only the simple stability region of the integral gain is shown in the special proportional tuning case for simplicity because to obtain the solution of integral gain is difficult.

Here, a digital IP control system for a second-order controlled object with lag time L without sensor dynamics is assumed. For simplicity, only special proportional gain case is shown.

\section{Transfer functions:}

$$
G(s)=\frac{K e^{-L s}}{T s+1}=\frac{K(1-0.5 L s)}{(T s+1)(0.5 L s+1)}=\frac{K \omega_{n}^{2}(1-0.5 L s)}{s^{2}+2 \varsigma \omega_{n} s+\omega_{n}^{2}}
$$




$$
\begin{gathered}
\omega_{n}=\frac{1}{\sqrt{0.5 T L}}, \varsigma=0.5 \sqrt{0.5 T L}\{(T+0.5 L) /(0.5 T L)\} \\
H(s)=\frac{K_{s}}{\varepsilon s+1}
\end{gathered}
$$

\section{Normalized operation:}

The normalize operations as same as above mentioned are done as follows.

$$
\begin{gathered}
\bar{s} \triangleq \frac{s}{\omega_{n}}, \bar{L} \triangleq L \omega_{n} \\
\bar{G}(\bar{s})=\frac{(1-0.5 \bar{L} \bar{s})}{\bar{s}^{2}+2 \varsigma \bar{s}+1} \\
\bar{\varepsilon} \triangleq \varepsilon \omega_{n} \\
\bar{H}(s)=\frac{1}{\overline{\varepsilon \bar{s}}+1} \\
\overline{K_{i} \triangleq} \frac{K_{i}}{\omega_{n}} \quad \frac{1}{\omega_{n}} \bar{p} \\
C(\bar{s})=\bar{K}_{i}\left(\frac{1}{\bar{s}}+\bar{p}\right) \quad C(s)=\bar{K}_{i} \omega_{n}\left(\frac{1}{s}+\frac{1}{\omega_{n}} \bar{p}\right)
\end{gathered}
$$

\section{Closed loop transfer function:}

The closed loop transfer function is obtained using above normalization as follows;

$$
\begin{aligned}
W(\bar{s})= & \frac{\bar{K}_{i}\left(\frac{1}{\bar{s}}+\bar{p}\right) \frac{(1-0.5 \bar{L} \bar{s})}{\left(\bar{s}^{2}+2 \varsigma \bar{s}+1\right)}}{1+\bar{K}_{i}\left(\frac{1}{\bar{s}}+\bar{p}\right) \frac{(1-0.5 \bar{L} \bar{s})}{(\overline{\varepsilon s}+1)\left(\bar{s}^{2}+2 \varsigma \bar{s}+1\right)}} \\
= & \frac{\bar{K}_{i}(1+\overline{p s})(1-0.5 \bar{L} \bar{s})(\bar{\varepsilon}+1)}{\overline{\varepsilon s}^{4}+(2 \bar{\varepsilon}+1) \bar{s}^{3}+\left(\bar{\varepsilon}+2 \varsigma-0.5 \bar{p} \bar{L} \bar{K}_{i}\right) \bar{s}^{2}+\left(1+\bar{K}_{i} \bar{p}-0.5 \bar{L} \bar{K}_{i}\right) \bar{s}+\bar{K}_{i}} \\
& \text { if } \quad \text { then } \\
& \text { if } \quad \bar{p}(\bar{s})=\frac{K_{i}(1-0.5 \bar{L} \bar{s})(\overline{\varepsilon s}+1)}{\overline{\varepsilon s} \bar{s}^{4}+(2 \varsigma \bar{\varepsilon}+1) \bar{s}^{3}+\left(\bar{\varepsilon}+2 \varsigma-0.5^{2} L^{2} \bar{K}_{i}\right) \bar{s}^{2}+\bar{s}+\bar{K}_{i}} \\
& W(\bar{s})=\frac{\text { then } \quad \bar{K}_{i}(1-0.5 \bar{L} \bar{s})(\bar{\varepsilon} \bar{s}+1)}{\bar{\varepsilon} \bar{s}^{4}+(2 \varsigma \bar{\varepsilon}+1) \bar{s}^{3}+(\bar{\varepsilon}+2 \varsigma) \bar{s}^{2}+\left(1-0.5 \bar{L} \bar{K}_{i}\right) \bar{s}^{2}+\bar{K}_{i}}
\end{aligned}
$$


Stability analysis by Hurwits Approach

1. $\bar{p}<0.5 \bar{L}, 0<\bar{K}_{i}<\min \left\{\frac{\bar{\varepsilon}+2 \varsigma}{0.5 \bar{p} \bar{L}}, \frac{1}{(0.5 \bar{L}-\bar{p})}\right\}, \varsigma>0, \bar{\varepsilon}>0$

$$
\begin{gathered}
\left\{(2 \varsigma \bar{\varepsilon}+1)\left(2 \varsigma+\bar{\varepsilon}-0.5^{2} \bar{L}^{2} \bar{K}_{i}\right)-\bar{\varepsilon}\right\}>\bar{K}_{i}(2 \varsigma \bar{\varepsilon}+1)^{2} \quad \text { when } \quad \bar{p}=0.5 \bar{L} \\
\frac{2 \varsigma\left(\bar{\varepsilon}^{2}+2 \varsigma \bar{\varepsilon}+1\right)}{(2 \varsigma \bar{\varepsilon}+1)\left\{(2 \varsigma \bar{\varepsilon}+1)+0.5^{2} \bar{L}^{2}\right\}}>\bar{K}_{i} \quad \text { when } \quad \bar{p}=0.5 \bar{L}
\end{gathered}
$$

$\mathrm{k}_{3}<\mathrm{k}_{2}$ then

$$
0<\bar{K}_{i}<\min \left\{\frac{\bar{\varepsilon}+2 \varsigma}{0.5^{2} \bar{L}^{2}}, \frac{2 \varsigma\left(\bar{\varepsilon}^{2}+2 \varsigma \bar{\varepsilon}+1\right)}{(2 \varsigma \bar{\varepsilon}+1)\left\{(2 \varsigma \bar{\varepsilon}+1)+0.5^{2} \bar{L}^{2}\right\}}\right\} \quad \text { when } \quad \bar{p}=0.5 \bar{L}
$$

In continuous region with one order modelling error,

$$
0<\bar{K}_{i}<\frac{2 \varsigma}{\left(1+0.5^{2} \bar{L}^{2}\right)} \quad \text { when } \quad \bar{p}=0.5 \bar{L}
$$

Analytical solution of Ki for flat gain curve using Stationary Points Investing for Fraction Equation approach is complicated to obtain, then it is remained for reader's theme. In the future, another approach will be developed for safe and simple robust control.

B. Simple soft M/A station

In this section, a configuration of simple soft M/A station and the feedback control system
with the station is shown for a simple safe interlock avoiding dangerous large overshoot.

B.1 Function and configuration of simple soft M/A station

This appendix describes a simple interlock plan for an simple soft M/A station that has a parameter-identification mode (manual mode) and a control mode (automatic mode).

The simple soft M/A station is switched from automatic operation mode to manual operation mode for safety when it is used to switch the identification mode and the control mode and when the value of $P v$ exceeds the prescribed range. This serves to protect the plant; for example, in the former case, it operates when the integrator of the PID controller varies erratically and the control system malfunctions. In the latter case, it operates when switching from $\mathrm{P}$ control with a large steady-state deviation with a high load to PI or PID control, so that the liquid in the tank spillovers. Other dangerous situations are not considered here because they do not fall under general basic control.

There have several attempts to arrange and classify the control logic by using a case base. Therefore, the M/A interlock should be enhanced to improve safety and maintainability; this has not yet been achieved for a simple M/ A interlock plan (Fig. A1).

For safety reasons, automatic operation mode must not be used when changing into manual operation mode by changing the one process value, even if the process value recovers to an appropriate level for automatic operation.

Semiautomatic parameter identification and PID control are driven by case-based data for memory of tuners, which have a nest structure for identification.

This case-based data memory method can be used for reusing information, and preserving integrity and maintainability for semiautomatic identification and control. The semiautomatic approach is adopted not only to make operation easier but also to enhance safety relative to the fully automatic approach. 
Notation in computer control (Fig. B1, B3)

Pv : Process value

Av: Actual value

$\mathrm{Cv}$ : Control value

Mv : Manipulated value

Sp: Set point

A : Auto

$\mathrm{M}:$ Manual

$\mathrm{T}:$ Test

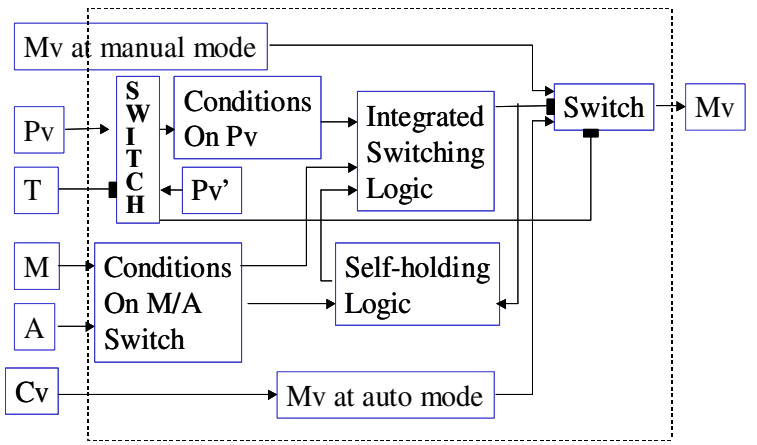

Fig. B1 A Configuration of Simple Soft M/A Station

B.2 Example of a SISO system

Fig. B2 shows the way of using M/A station in a configuration of a SISO control system.

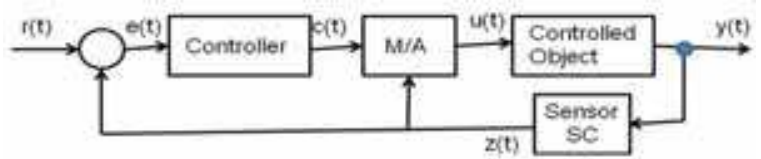

Fig. B2 Configuration of a IP Control System with a M/A Station for a SISO Controlled Object where the transfer function needed in Fig.B2 is as follows.

1. Controlled Object: $G(s)=\frac{K}{T s+1} e^{-L s}$

2. Sensor \& Signal Conditioner: $G_{s}(s)=\frac{K_{s}}{T_{s} s+1}$

3. Controller: $C(s)=0.5 K_{i 2}\left(\frac{1}{s}+0.5 L\right)$

4. Sensor Caribration Gain: $1 / K_{s}$

5. Normalized Gain before M/A Station: $1 / \sqrt{0.5 T L}$

6. Normalized Gain after M/A Station: $1 / K$

Fig. B3 shows examples of simulated results for 2 kinds of switching mode when $P v$ becomes higher than a given threshold. (a) shows one to out of service and (b) does to manual mode.

In former, $\mathrm{Mv}$ is down and $\mathrm{Cv}$ is almost hold. In latter, $\mathrm{Mv}$ is hold and $\mathrm{Cv}$ is down. 


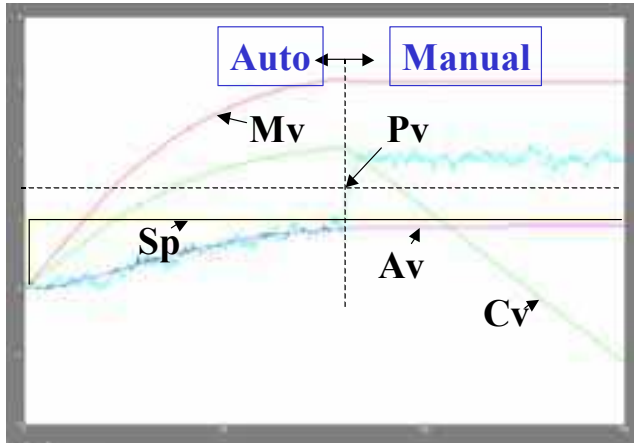

(a) Switching example from auto mode to out of service by Pv High

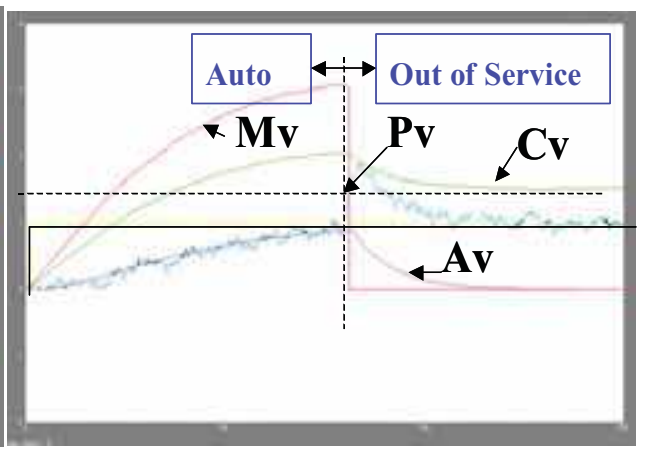

(b) Switching example from auto mode to manual mode by Pv High

Fig. B3 Simulation results for 2 kinds of switching mode

C. New norm and expansion of small gain theorem

In this section, a new range restricted norm of Handy space with window(Kohonen T., 1995) $H_{\infty}^{w}$ is defined for I, of which window is described to notation of norm with superscript $w$, and a new expansion of small gain theorem based on closed loop system like general $H_{\infty}^{w}$ control problems and robust sensitivity analysis is shown for applying the robust PI control concept of this chapter to MIMO systems.

The robust control was aims soft servo and requested internal stability for a closed loop control system. Then, it was difficult to apply process control systems or hard servo systems which was needed strong robust stability without deviation from the reference value in the steady state like integral terms.

The method which sets the maximum value of closed loop gain curve to 1 and the results of this numerical experiments indicated the above sections will imply the following new expansion of small gain theorem which indicates the upper limit of Hardy space norm of a forward element using the upper limit of all uncertain feedback elements for robust stability.

For the purpose using unbounded functions in the all real domain on frequency like integral term in the forward element, the domain of Hardy norm of the function concerned on frequency is limited clearly to a section in a positive real one-order space so that the function becomes bounded in the section.

\section{Proposition}

Assuming that feedback transfer function $\mathrm{H}(\mathrm{s})$ (with uncertainty) are stable and the following inequality is holds,

$$
\|H(s)\|_{\infty} \leq \frac{1}{\gamma}, \gamma \geq 1
$$

Moreover, if the negative closed loop system as shown in Fig.C-1 is stable and the following inequality holds,

$$
\|W(s)\|_{\infty}=\left\|\frac{G(s)}{1+G(s) H(s)}\right\|_{\infty} \leq 1
$$


then the following inequality on the open loop transfer function is hold in a region of frequency.

$$
\|G(j \omega) H(j \omega)\|_{\infty}^{w} \leq \frac{1}{\gamma-1}, \gamma \geq 1 \quad \text { for } \quad \omega \in\left[\omega_{\min }, \omega_{\max }\right]
$$

In the same feedback system, G(s) holds the following inequality in a region of frequency.

$$
\|G(j \omega)\|_{\infty}^{w} \leq \frac{\gamma}{\gamma-1}, \gamma \geq 1 \quad \text { for } \quad \omega \in\left[\omega_{\min }, \omega_{\max }\right]
$$

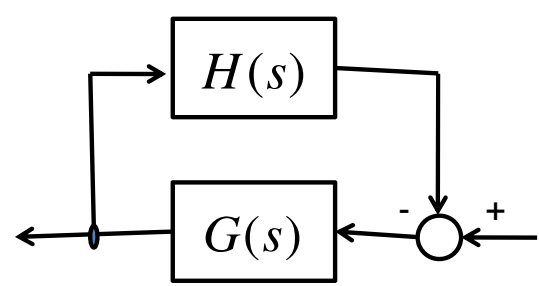

Fig. C-1 Configuration of a negative feed back system

(proof)

Using triangle inequality on separation of norms of summension and inequality on separation of norms of product like Helder's one under a region of frequency $\left[\omega_{\min }, \omega_{\max }\right]$, as a domain of the norm of Hardy space with window, the following inequality on the frequency transfer function of $G(j \omega)$ is obtained from the assumption of the proposition.

$$
\begin{gathered}
\|W(j \omega)\|_{\infty}=\left\|\frac{G(j \omega)}{1+G(j \omega) H(j \omega)}\right\|_{\infty} \leq 1 \\
\|G(j \omega)\|_{\infty}^{w} \leq\|1+G(j \omega) H(j \omega)\|_{\infty}^{w} \leq 1+\|G(j \omega)\|_{\infty}^{w}\|H(j \omega)\|_{\infty}^{w} \\
1-\|H(j \omega)\|_{\infty}^{w} \leq\left\|\frac{1}{G(j \omega)}\right\|_{\infty}^{w} \\
\text { if }\|H(j \omega)\|_{\infty}^{w} \leq \frac{1}{\gamma} \leq 1, \gamma \geq 1 \text { then } \\
\frac{\gamma}{\gamma-1} \geq \frac{1}{1-\|H(j \omega)\|_{\infty}^{w}} \geq\|G(j \omega)\|_{\infty}^{w}
\end{gathered}
$$

Moreover, the following inequality on open loop frequency transfer function is shown.

$$
\frac{1}{\gamma-1} \geq\|G(j \omega)\|_{\infty}^{w}\|H(j \omega)\|_{\infty}^{w} \geq\|G(j \omega) H(j \omega)\|_{\infty}^{w}
$$

On the inequality of norm, the reverse proposition may be shown though the separation of product of norms in the Hardy space with window are not clear. The sufficient conditions on closed loop stability are not clear. They will remain reader's theme in the future. 
D. Parametric robust topics

In this section, the following three topics (Bhattacharyya S. P., Chapellat H., and Keel L. H., 1994.) are introduced at first for parametric robust property in static one, dynamic one and stable one as assumptions after linearizing a class of non-linear system to a quasi linear parametric variable $(Q L P V)$ model by Taylar expansion using first order reminder term. (M.Katoh, 2010)

1. Continuity for change of parameter

\section{Boundary Crossing Theorem}

1) fixed order polynomials $P(\lambda, s)$

2) continuous polynomials with respect to one parameter $\lambda$ on a fixed interval $I=[a, b]$.

If $\mathrm{P}(\mathrm{a}, \mathrm{s})$ has all its roots in $\mathrm{S}, \mathrm{P}(\mathrm{b}, \mathrm{s})$ has at least one root in $\mathrm{U}$, then there exists at least one $\rho$ in $(a, b]$ such that:

a) $P(\rho, s)$ has all roots in $S$ UDS

b) $P(\rho, s)$ has at least one root in $\partial S$

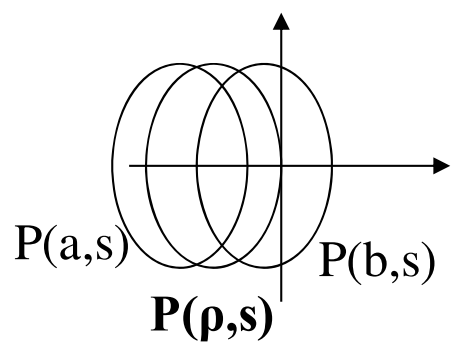

Fig. D-1 Image of boundary crossing theorem

2. Convex for change of parameter

Segment Stable Lemma

Let define a segment using two stable polynomials as follows.

$$
\begin{aligned}
& \delta_{\lambda}(s) \triangleq \lambda \delta_{1}(s)+(1-\lambda) \delta_{2}(s) \\
& {\left[\delta_{1}(s), \delta_{2}(s)\right] \triangleq\left\{\delta_{\lambda}(s): \lambda \in[0,1]\right\}} \\
& \text { where } \delta_{1}(s), \delta_{2}(s) \_i s_{-} \text {polynomials_of_deg ree_n } \\
& \quad \text { and_stable_with_respect_to_ } s
\end{aligned}
$$

Then, the followings are equivalent:

a) The segment $\left[\delta_{1}(s), \delta_{2}(s)\right]$ is stable with respect to $S$

$$
\text { b) } \delta_{\lambda}\left(s^{*}\right) \neq 0, \quad \text { for_all_ } s^{*} \in \partial S ; \quad \lambda \in[0,1]
$$

3. Worst stability margin for change of parameter

Parametric stability margin (PSM) is defined as the worst case stability margin within the parameter variation. It can be applied to a QLPV system of a class of non-linear system. There are non-linear systems such as becoming worse stability margin than linearized system although there are ones with better stability margin than it. There is a case which is characterized by the one parameter $m$ which describes the injection rate of $\mathrm{I} / \mathrm{O}$, the interpolation rate of segment or degree of non-linearity.

E. Risk and Merit Analysis

Let show a summary and enhancing of the risk discussed before sections for safety in the following table. 


\begin{tabular}{|l|l|l|}
\hline \multicolumn{1}{|c|}{ Kinds } & \multicolumn{1}{|c|}{ Evaluation of influence } & \multicolumn{1}{c|}{ Countermeasure } \\
\hline $\begin{array}{l}\text { 1) Disconnection of } \\
\text { feedback line }\end{array}$ & $\begin{array}{l}\text { 1) Spill-over threshold } \\
\text { 2) Overshoot over limit } \\
\text { value }\end{array}$ & $\begin{array}{l}\text { Auto change to manual } \\
\text { mode by M/A station } \\
\text { Auto shut down }\end{array}$ \\
\hline $\begin{array}{l}\text { Change of tuning region } \\
\text { from IPS to IPL by making } \\
\text { proportional gain to large }\end{array}$ & $\begin{array}{l}\text { Grade down of stability } \\
\text { region from strong or weak } \\
\text { to weak or un-stability }\end{array}$ & $\begin{array}{l}\text { Use IP0 and not use IPS } \\
\text { Not making proportional } \\
\text { gain to large in IPS tuning } \\
\text { region }\end{array}$ \\
\hline $\begin{array}{l}\text { Change of damping } \\
\text { coefficient or inverse of } \\
\text { time constant over weak } \\
\text { robust limit }\end{array}$ & $\begin{array}{l}\text { Grade down of stability } \\
\text { region from strong or weak } \\
\text { to weak or un-stability }\end{array}$ & $\begin{array}{l}\text { Change of tuning region } \\
\text { from IPL to IPS or IP0 }\end{array}$ \\
\hline
\end{tabular}

Table E-1 Risk analysis for safety

It is important to reduce risk as above each one by adequate countermeasures after understanding the property of and the influence for the controlled objects enough.

Next, let show a summary and enhancing of the merit and demerit discussed before sections for robust control in the following table, too.

\begin{tabular}{|c|c|c|}
\hline Kinds & Merit & Demerit \\
\hline $\begin{array}{l}\text { 1) Steady state error is } \\
\text { vanishing as time by effect } \\
\text { of integral }\end{array}$ & $\begin{array}{l}\text { 1) It is important property } \\
\text { in process control and hard } \\
\text { servo area }\end{array}$ & $\begin{array}{l}\text { It is dislike property in soft } \\
\text { servo and robot control } \\
\text { because of hardness for } \\
\text { disturbance }\end{array}$ \\
\hline $\begin{array}{l}\text { There is a strong robust } \\
\text { stability damping region in } \\
\text { which the closed loop gain } \\
\text { margin for any uncertainty } \\
\text { is over } 2 \text { and almost not } \\
\text { changing. }\end{array}$ & $\begin{array}{l}\text { It is uniform safety for } \\
\text { some proportional gain } \\
\text { tuning region and changing } \\
\text { of damping coefficient. } \\
\text { For integral loop gain } \\
\text { tuning, it recommends the } \\
\text { simple limiting sensitivity } \\
\text { approach. }\end{array}$ & $\begin{array}{l}\text { 1) Because the region is } \\
\text { different by proportional } \\
\text { gain, there is a risk of grade } \\
\text { down by the gain tuning. }\end{array}$ \\
\hline $\begin{array}{l}\text { There is a weak robust } \\
\text { stability damping region in } \\
\text { which the worst closed loop } \\
\text { gain margin for any } \\
\text { uncertainty is over given } \\
\text { constant. }\end{array}$ & $\begin{array}{l}\text { 1) It can specify the grade } \\
\text { of robust stability for any } \\
\text { uncertainty }\end{array}$ & $\begin{array}{l}\text { 1) Because the region is } \\
\text { different by proportional } \\
\text { gain, there is a risk of grade } \\
\text { down by the gain tuning. } \\
\text { It is different safety for } \\
\text { some proportional gain } \\
\text { tuning region. }\end{array}$ \\
\hline
\end{tabular}

Table E-2 Merit analysis for control

It is important to apply to the best application area which the merit can be made and the demerit can be controlled by the wisdom of everyone. 


\section{References}

Bhattacharyya S. P., Chapellat H., and Keel L. H.(1994). Robust Control, The Parametric Approach, Upper Saddle River NJ07458 in USA: Prentice Hall Inc.

Katoh M. and Hasegawa H., (1998). Tuning Methods of 2nd Order Servo by I-PD Control Scheme, Proceedings of The 41st Joint Automatic Control Conference, pp. 111-112. (in Japanese)

Katoh M.,(2003). An Integral Design of A Sampled and Continuous Robust Proper Compensator, Proceedings of CCCT2003, (pdf000564), Vol. III, pp. 226-229.

Katoh M.,(2008). Simple Robust Normalized IP Control Design for Unknown Input Disturbance, SICE Annual Conference 2008, August 20-22, The University ElectroCommunication, Japan, pp.2871-2876, No.:PR0001/08/0000-2871

Katoh M., (2009). Loop Gain Margin in Simple Robust Normalized IP Control for Uncertain Parameter of One-Order Model Error, International Journal of Advanced Computer Engineering, Vol.2, No.1, January-June, pp.25-31, ISSN:0974-5785, Serials Publications, New Delhi (India)

Katoh M and Imura N., (2009). Double-agent Convoying Scenario Changeable by an Emergent Trigger, Proceedings of the $4^{\text {th }}$ International Conference on Autonomous Robots and Agents, Feb 10-12, Wellington, New Zealand, pp.442-446

Katoh M. and Fujiwara A., (2010). Simple Robust Stability for PID Control System of an Adjusted System with One-Changeable Parameter and Auto Tuning, International Journal of Advanced Computer Engineering, Vol.3, No.1, ISSN:0974-5785, Serials Publications, New Delhi (India)

Katoh M.,(2010). Static and Dynamic Robust Parameters and PI Control Tuning of TV-MITE Model for Controlling the Liquid Level in a Single Tank", TC01-2, SICE Annual Conference 2010, 18/ August TC01-3

Krajewski W., Lepschy A., and Viaro U.,(2004). Designing PI Controllers for Robust Stability and Performance, Institute of Electric and Electronic Engineers Transactions on Control System Technology, Vol. 12, No. 6, pp. 973- 983.

Kohonen T.,(1995, 1997). Self-Organizing Maps, Springer

Kojori H. A., Lavers J. D., and Dewan S. B.,(1993). A Critical Assessment of the ContinuousSystem Approximate Methods for the Stability Analysis of a Sampled Data System, Institute of Electric and Electronic Engineers Transactions on Power Electronics, Vol. 8, No. 1, pp. 76-84.

Miyamoto S.,(1998). Design of PID Controllers Based on $H_{\infty}$-Loop Shaping Method and LMI Optimization, Transactions of the Society of Instrument and Control Engineers, Vol. 34, No. 7, pp. 653-659. (in Japanese)

Namba R., Yamamoto T., and Kaneda M., (1998). A Design Scheme of Discrete Robust PID Control Systems and Its Application, Transactions on Electrical and Electronic Engineering, Vol. 118-C, No. 3, pp. 320-325. (in Japanese)

Olbrot A. W. and Nikodem M.,(1994) . Robust Stabilization: Some Extensions of the Gain Margin Maximization Problem, Institute of Electric and Electronic Engineers Transactions on Automatic Control, Vol. 39, No. 3, pp. 652- 657.

Zbou K. with Doyle F. C. and Glover K.,(1996). Robust and Optimal Control, Prentice Hall Inc.

Zhau K. and Khargonekar P.P., (1988). An Algebraic Riccati Equation Approach to H Optimization, Systems \& Control Letters, 11, pp.85-91. 


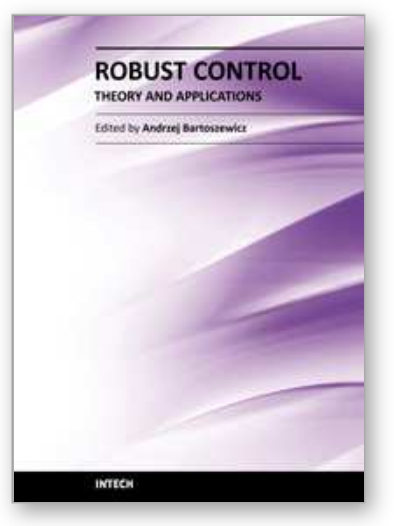

\author{
Robust Control, Theory and Applications \\ Edited by Prof. Andrzej Bartoszewicz
}

ISBN 978-953-307-229-6

Hard cover, 678 pages

Publisher InTech

Published online 11, April, 2011

Published in print edition April, 2011

The main objective of this monograph is to present a broad range of well worked out, recent theoretical and application studies in the field of robust control system analysis and design. The contributions presented here include but are not limited to robust PID, H-infinity, sliding mode, fault tolerant, fuzzy and QFT based control systems. They advance the current progress in the field, and motivate and encourage new ideas and solutions in the robust control area.

\title{
How to reference
}

In order to correctly reference this scholarly work, feel free to copy and paste the following:

Makoto Katoh (2011). Simple Robust Normalized PI Control for Controlled Objects with One-order Modelling Error, Robust Control, Theory and Applications, Prof. Andrzej Bartoszewicz (Ed.), ISBN: 978-953-307-229-6, InTech, Available from: http://www.intechopen.com/books/robust-control-theory-and-applications/simplerobust-normalized-pi-control-for-controlled-objects-with-one-order-modelling-error

\section{INTECH}

open science | open minds

\author{
InTech Europe \\ University Campus STeP Ri \\ Slavka Krautzeka 83/A \\ 51000 Rijeka, Croatia \\ Phone: +385 (51) 770447 \\ Fax: +385 (51) 686166 \\ www.intechopen.com
}

\author{
InTech China \\ Unit 405, Office Block, Hotel Equatorial Shanghai \\ No.65, Yan An Road (West), Shanghai, 200040, China \\ 中国上海市延安西路65号上海国际贵都大饭店办公楼 405 单元 \\ Phone: +86-21-62489820 \\ Fax: $+86-21-62489821$
}


(C) 2011 The Author(s). Licensee IntechOpen. This chapter is distributed under the terms of the Creative Commons Attribution-NonCommercialShareAlike-3.0 License, which permits use, distribution and reproduction for non-commercial purposes, provided the original is properly cited and derivative works building on this content are distributed under the same license. 